
\title{
3 Research Square \\ Evaluation of an Oral Sodium Bicarbonate Protocol for High-Dose Methotrexate Urine Alkalinization
}

\author{
Rachel Heisler ( $\nabla$ rheisler@ualberta.ca ) \\ University of Alberta Hospital https://orcid.org/0000-0001-5577-8854 \\ Jordan Kelly \\ Alberta Health Services
}

Sara Abedinzadegan Abdi

Peter Lougheed Centre

Jennifer Hawker

Foothills Medical Centre

Leanne Fong

Foothills Medical Centre

Janet Quon

Peter Lougheed Centre

Josee Rioux

Victoria General Hospital: Queen Elizabeth II Health Sciences Centre

Jennifer Jupp

Alberta Health Services

\section{Research Article}

Keywords: high dose methotrexate, sodium bicarbonate, oral, urine alkalinization, supportive care

Posted Date: April 16th, 2021

DOI: https://doi.org/10.21203/rs.3.rs-328004/v1

License: (c) (1) This work is licensed under a Creative Commons Attribution 4.0 International License.

Read Full License

Version of Record: A version of this preprint was published at Supportive Care in Cancer on September 1st, 2021. See the published version at https://doi.org/10.1007/s00520-021-06503-3. 


\section{Abstract}

Purpose: Intravenous (IV) sodium bicarbonate is considered standard therapy for high-dose methotrexate (HDMTX) urine alkalinization. Due to a national IV sodium bicarbonate shortage, an oral (PO) sodium bicarbonate protocol was implemented by Alberta Health Services (AHS) for HDMTX urine alkalinization. This study aims to evaluate the efficacy and safety of the PO sodium bicarbonate protocol compared to IV sodium bicarbonate for HDMTX urine alkalinization.

Methods: A retrospective chart review of adult patients who received HDMTX $\left(>500 \mathrm{mg} / \mathrm{m}^{2}\right)$ with sodium bicarbonate for urine alkalinization at 4 hospitals in Alberta was conducted. Patients who received IV sodium bicarbonate between January-June 2017 and PO sodium bicarbonate between July-December 2017 were compared for the primary outcome of time to methotrexate clearance.

Results: A total of 84 and 78 HDMTX cycles were included in the IV and PO cohorts, respectively. No difference in time to methotrexate clearance was seen between the IV and PO cohorts, $91.6( \pm 35.4)$ hours and 95.2 ( \pm 44 ) hours respectively; $p=0.5$. The proportion of HDMTX cycles that experienced $a>25 \%$ increase in serum creatinine was not statistically significant, IV protocol $12 \%$ and PO protocol $5 \% ; p=0.13$. Nausea and emesis occurred more frequently in the PO cohort than the IV cohort, though rarely resulted in refused doses or change to alternate sodium bicarbonate formulations.

Conclusions: The results of this study indicate that the AHS PO sodium bicarbonate protocol was no different in time to methotrexate clearance or rates of increased serum creatinine when compared to IV sodium bicarbonate.

\section{Background}

Methotrexate (MTX) is a folate antimetabolite used at high doses $\left(>500 \mathrm{mg} / \mathrm{m}^{2}\right)$ to treat oncologic diagnoses such as leukemia, lymphoma, and osteosarcoma. Along with its therapeutic benefit, high-dose methotrexate (HDMTX) exposure is associated with potentially serious adverse effects.

MTX and its metabolites can precipitate in the renal tubules leading to nephrotoxicity in $2-12 \%$ of patients [1]. As MTX is primarily renally eliminated, this nephrotoxicity can lead to delayed methotrexate clearance and higher rates of additional adverse effects such as mucositis, myelosuppression, and hepatotoxicity $[1,2]$. MTX has poor solubility in acidic environments and when the urine $\mathrm{pH}$ is 7 or higher, solubility is greatly improved [2]. As such, it is standard practice that patients receiving HDMTX undergo vigorous hydration and urine alkalinization with intravenous (IV) sodium bicarbonate to maintain a urine $\mathrm{pH}$ above 7 [1-3]. At Alberta Health Services (AHS) these supportive care measures are routinely used in all patients receiving HDMTX.

In 2017, a North American wide shortage of IV sodium bicarbonate necessitated the development of an alternate method for HDMTX urine alkalinization. Sodium acetate is a common alternative to sodium bicarbonate [4], however, a concomitant shortage of IV sodium acetate restricted its use. Acetazolamide 
is an inhibitor of carbonic anhydrase and causes urinary alkalinization by increasing the renal excretion of sodium bicarbonate [5]. Data supporting the use of acetazolamide as monotherapy for HDMTX urine alkalinization is limited to a small case series [6]. Additionally, concerns regarding adverse effects such as metabolic acidosis and possible loss of the urine alkalinizing effect with prolonged administration limits its use [6, 7]. Since enteral sodium bicarbonate formulations were unaffected by the shortage and their routine use has demonstrated sustained urine pH over 7 [8], AHS developed an oral (PO) sodium bicarbonate protocol extrapolated from the Johns Hopkins Hospital protocol (see Appendix). The PO protocol utilizes routine PO sodium bicarbonate in all patients treated with HDMTX, with parenteral sodium bicarbonate being restricted to patients not tolerating, or unable to achieve a target urine $\mathrm{pH}$ with PO administration. Additionally, the use of lactated Ringer's (LR), which converts to bicarbonate in vivo, provides another bicarbonate source.

With future drug shortages remaining a concern, it has become increasingly important that alternate HDMTX urine alkalinization methods are reviewed to ensure appropriate use. Therefore, our study was developed to review the efficacy and safety of the AHS PO sodium bicarbonate protocol compared to a historical cohort utilizing IV sodium bicarbonate for HDMTX urine alkalinization.

\section{Methods}

Study Design

A retrospective chart review was conducted at four acute care centers in Alberta, Canada: the Foothills Medical Centre, Peter Lougheed Centre, Cross Cancer Institute, and University of Alberta Hospital. Adult patients that received HDMTX ( $>500 \mathrm{mg} / \mathrm{m}^{2}$ ) with sodium bicarbonate for urine alkalinization were reviewed for study inclusion. The IV cohort included HDMTX cycles that utilized IV sodium bicarbonate for urine alkalinization between January and June, 2017. The PO cohort included HDMTX cycles that utilized the oral sodium bicarbonate protocol between July and December, 2017. HDMTX cycles were excluded if a mixed urine alkalinization protocol was administered (routine use of both PO and IV sodium bicarbonate during the same HDMTX cycle), data was incomplete, errors administrating protocol dosing occurred, alternate hydration protocols were used, or sodium acetate was administered. If a patient received multiple cycles of HDMTX, each cycle was included in the appropriate cohort provided inclusion criteria was met. Patients receiving HDMTX were identified by reviewing electronic chart records or pharmacy records utilized at each center. Data was collected from electronic and paper charts. Ethics approval was obtained from The Health Research Ethics Board of Alberta Cancer Committee (HREBA-CC). Due to the retrospective nature of the study, a waiver of consent was obtained. All data was collected anonymously and presented aggregately.

\section{$\underline{\text { Outcomes }}$}

The primary outcome of the study was time from HDMTX administration to MTX clearance. MTX clearance was defined as the first of three possible endpoints: MTX level $\leq 0.05 \mu \mathrm{mol} / \mathrm{L}$, first dose of 
leucovorin $5 \mathrm{mg}$, or discharge.

Secondary outcomes included the time to MTX clearance for each course of HDMTX, number of sodium bicarbonate bolus doses administered, total dose of sodium bicarbonate administered for each HDMTX cycle, number of protocol leucovorin doses required to achieve MTX clearance, proportion of patients with a $>25 \%$ increase in serum creatinine after MTX clearance, the proportion of HDMTX cycles with adverse events (mucositis, nausea, emesis, diarrhea, neurologic complications and hypernatremia), and the proportion of HDMTX cycles in the PO cohort with documented administration issues such as refusal of doses or a need to change sodium bicarbonate formulation. Neurologic complications were defined as somnolence, confusion, and seizure. Diarrhea was defined as $\geq 4$ stools per day and hypernatremia was defined as serum sodium level $>145 \mathrm{mmol} / \mathrm{L}$. To assist with interpretation of the primary outcome, each HDMTX cycle was reviewed for concurrent administration of interacting medications that may impact time to MTX clearance.

A subgroup analysis was performed in patients included in both IV and PO cohorts for different HDMTX cycles to identify trends in time to MTX clearance and proportion of serum creatinine increases $>25 \%$, as patients acted as their own control.

\section{$\underline{\text { Treatments }}$}

Hydration and sodium bicarbonate therapy started the evening before HDMTX administration in both the IV and PO protocols. Patients in the IV cohort received IV sodium bicarbonate $100 \mathrm{mmol} / \mathrm{L}$ in D $5 \mathrm{~W}+20$ $\mathrm{mmol} / \mathrm{L}$ of potassium chloride at $200 \mathrm{~mL} / \mathrm{hr}$ until MTX clearance. Treating clinicians were free to adjust the infusion rate as needed. Patients in the PO cohort received sodium bicarbonate 1300 to $1500 \mathrm{mg}$ q6h and LR at $2 \mathrm{~mL} / \mathrm{kg} / \mathrm{hr}$ until MTX clearance. LR was chosen for hydration as it provides $28 \mathrm{mmol}$ of bicarbonate per liter. Patients received leucovorin as per the treatment protocol until MTX clearance. Once MTX had cleared, leucovorin dosing was decreased to $5 \mathrm{mg}$ PO four times daily for four more days.

Urine $\mathrm{pH}$ was measured prior to HDMTX administration and periodically after administration until MTX clearance. HDMTX was administered once urine $\mathrm{pH}$ reached target of $\geq 7$. If the urine $\mathrm{pH}$ was below 7 at any time, an IV bolus of $50 \mathrm{mmol}$ sodium bicarbonate could be administered in the IV cohort. Patients in the PO cohort received either an IV sodium bicarbonate $50 \mathrm{mmol}$ bolus or a $6500 \mathrm{mg}$ PO sodium bicarbonate bolus at the discretion of the treating physician. If the urine $\mathrm{pH}$ failed to reach target after two bolus doses in the PO cohort, the routine dose of sodium bicarbonate was escalated to $6500 \mathrm{mg} q 6 \mathrm{~h}$. Further escalation to $10000 \mathrm{mg}$ q6h occurred if the urine $\mathrm{pH}$ continued to be low after 2 bolus doses on routine dosing of $6500 \mathrm{mg} \mathrm{q6h}$ (Table 1). Frequency and timing of urine $\mathrm{pH}$ measurements was not standardized due to differences in center specific practices. MTX levels were monitored daily after HDMTX administration until a level of $\leq 0.05 \mu \mathrm{mol} / \mathrm{L}$ was achieved.

$\underline{\text { Statistical Analysis }}$ 
A convenience sample was used with data collected using RedCap and analyzed in Excel. Descriptive statistics were used to analyze both cohorts, including mean \pm SD and median (range). Comparisons between the IV and PO cohorts were calculated using students t-test and Chi-squared test for data with assumed normal distribution. Wilcoxon Rank-Sum test was used for comparison of data following nonparametric distribution.

\section{Results}

A total of 177 HDMTX cycles were reviewed for inclusion, of which 15 cycles were excluded for incomplete data $(n=6)$, use of a mixed protocol $(n=3)$, use of an alternate hydration protocol $(n=4)$, administration of sodium acetate $(n=1)$, and errors administering protocol dosing $(n=1)$. Therefore, 162 HDMTX cycles, in a total of 79 patients, were included in the study: 84 HDMTX cycles (40 patients) in the IV cohort and 78 HDMTX (44 patients) in the PO cohort (Figure 1). Five patients were included in both the IV and PO cohorts as they were administered HDMTX cycles that qualified for each cohort.

Baseline characteristics were similar between the two groups with the exception that significantly more patients in the PO cohort had an "other" diagnosis which included mantle cell lymphoma, follicular lymphoma, biphenotypic leukemia, plasmablastic lymphoma and peripheral T-cell lymphoma (Table 2).

\section{Time to Methotrexate Clearance}

No statistically significant difference in time to MTX clearance was seen between the IV and PO cohorts, 91.6 ( \pm 35.4 ) hours and $95.2( \pm 44)$ hours respectively; $p=0.5$. Both cohorts were similar in the proportion of cycles that met each endpoint defining MTX clearance (Table 3). When analyzed based on increasing number of HDMTX courses, there was no difference in time to MTX clearance between the IV and PO cohorts, nor were there any trends in time to MTX clearance identified (data not shown).

\section{Sodium Bicarbonate, Fluid and Leucovorin Doses}

A total of 21 sodium bicarbonate bolus doses were given for low urine $\mathrm{pH}$ during 14 (17\%) different HDMTX cycles in the IV cohort and 105 sodium bicarbonate bolus doses were given during 37 (47\%) different HDMTX cycles in the PO cohort; $\mathrm{p}=<0.01$. Of the bolus doses given in the PO cohort, 12 were administered as an IV bolus. Notably, the mean total dose of sodium bicarbonate administered from all sources during each cycle of HDMTX was significantly lower in the PO cohort: $992( \pm 432) \mathrm{mmol}$, compared to the IV cohort: $1984( \pm 850) \mathrm{mmol}, \mathrm{p}=<0.01$. The average volume of fluid administered during each HDMTX cycle in the IV cohort was $19692( \pm 8574) \mathrm{mL}$ and $17548( \pm 7574) \mathrm{mL}$ in the PO cohort; $p=0.09$. There was no difference in the number of leucovorin doses given prior to MTX clearance between the two cohorts (Table 4).

\section{$\underline{\text { Adverse Events }}$}

Compared to baseline serum creatinine values, 10 (12\%) and 4 (5\%) HDMTX cycles experienced a greater than $25 \%$ increase in serum creatinine after methotrexate clearance in the IV cohort and PO cohort, 
respectively; $p=0.13$.

A total of 29 HDMTX cycles experienced at least 1 adverse event in the IV cohort and 43 HDMTX cycles in the PO cohort, $p=<0.01$. Rates of specific adverse events were similar between the two groups apart from nausea and emesis, which occurred more often in the PO cohort (Table 5).

There were no patients in the PO cohort that required switching from routine PO sodium bicarbonate to IV sodium bicarbonate due to intolerance. Administration issues were identified in 2 HDMTX cycles in the PO cohort. Patient refusal to take a PO dose secondary to severe nausea was documented during one HDMTX cycle. Another HDMTX cycle documented a switch from sodium bicarbonate tablets to sodium citrate-citric acid liquid due to difficulties swallowing tablets.

\section{$\underline{\text { Interacting Medications }}$}

Overall, a total of 15 (18\%) HDMTX cycles in the IV cohort and 12 (15\%) HDMTX cycles in the PO cohort received at least 1 interacting medication; $p=0.67$. There were no HDMTX cycles in either cohort that received proton pump inhibitors or non-steroidal anti-inflammatory medications other than low dose acetylsalicylic acid. Both cohorts had a similar proportion of cycles that received concomitant interacting antibiotics (penicillin, amoxicillin-clavulanate, ampicillin, cloxacillin, piperacillin-tazobactam, tetracycline, ciprofloxacin, doxycycline and sulfonamides); IV cohort: 9 (11\%), PO cohort: 7 (9\%), p=0.9. Concomitant administration of other interacting medications (amiodarone, cyclosporine A, probenecid, gemfibrozil, levetiracetam, procarbazine, and sulfasalazine) were similar between the IV and PO cohorts; IV cohort: 10 (12\%), PO cohort: 5 (6\%), $p=0.23$.

\section{Subgroup Analysis of Patients Included in Both IV and PO Cohorts}

Five patients were included in both the IV and PO cohorts. No trends in time to MTX clearance, nor the number of cycles that experienced a greater than $25 \%$ increase in serum creatinine, could be identified comparing the IV and PO protocols in these 5 patients (data not shown).

\section{Discussion}

The results of this study demonstrate that the AHS PO sodium bicarbonate is comparable to our historical IV sodium bicarbonate protocol for HDMTX urine alkalinization. Our results align with that of other oral HDMTX urine alkalinization regimens previously published. A single-center, retrospective chart review by Roy et al. compared IV sodium bicarbonate to scheduled sodium bicarbonate $3250 \mathrm{mg} \mathrm{PO} \mathrm{q2h}$ plus as needed acetazolamide for low urine $\mathrm{pH}$ in adults receiving HDMTX [9]. Mean time to MTX clearance was found to be no different between the two cohorts; $64.2 \pm 45.3$ vs. $64.1 \pm 45.8$ hours, $p=N S$. Compared to our study, Roy et al. had a shorter time to MTX clearance. While they also used a higher PO sodium bicarbonate dose than our protocol, a higher MTX level of $0.1 \mu \mathrm{mol} / \mathrm{L}$ was used to define MTX clearance than our local standard and may explain their shorter time to MTX clearance. Another retrospective cohort study by Kramer et al. compared a predominantly enteral protocol with combination 
sodium bicarbonate and acetazolamide to a predominately parenteral protocol in adult patients receiving HDMTX [10]. Time to MTX clearance was not found to be statistically different between the enteral protocol, $98 \pm 38$ hours, and the parenteral protocol, $88 \pm 36$ hours; $p=0.06$. Of note, the starting dose of enteral sodium bicarbonate $3900 \mathrm{mg} \mathrm{PO}$ q6h in this protocol was higher than our PO protocol. Rouch, et al. published a retrospective cohort study also finding no difference in time to MTX clearance between HDMTX cycles utilizing enteral alkalinizing agents and those utilizing parenteral alkalinization agents [11]. The enteral and parenteral methods were not described, however.

An interesting finding in our study was that our PO cohort utilized nearly $50 \%$ less sodium bicarbonate per HDMTX cycle than the IV cohort. Despite the lower sodium bicarbonate dose, no differences in time to MTX clearance or the rates of increased serum creatinine were seen between the two cohorts. This finding of an overall lower enteral sodium bicarbonate dose being comparable to a higher IV sodium bicarbonate dose is reflected in the literature, both in the pediatric and adult populations [9-10, 12-13]. Consideration to whether lower IV sodium bicarbonate doses have comparable outcomes to those currently prescribed may be of interest.

A common concern with the use of PO sodium bicarbonate for HDMTX urine alkalinization is the administration burden as a high quantity of tablets or liquid may be needed. Using an oral sodium bicarbonate dose to mirror that provided by the standard IV protocol, Roy, et al. found that only $67.5 \%$ of expected daily PO sodium bicarbonate doses were received by patients [9]. For this reason, AHS chose to utilize a lower routine dose of PO sodium bicarbonate and LR for hydration to aid tolerability. Additional sodium bicarbonate bolus doses and escalation of the routine PO dose as needed, helped ensure patients could be managed on the lowest effective dose. An alternate method for HDMTX urine alkalinization is combination therapy with PO sodium bicarbonate and acetazolamide. Various protocols have been recently published utilizing this combination $[6,10,14]$. Acetazolamide use carries the risk of additional adverse effects such as metabolic acidosis and gastrointestinal intolerance. As well, it is also associated with an increased pill burden. The current literature for combination therapy uses a higher PO sodium bicarbonate dose than our protocol and therefore is not expected to have a more acceptable administration burden. With our protocol, we were able to successfully achieve MTX clearance using lower routine PO sodium bicarbonate dosing than that currently reported in the literature.

Similar to the results reported by Roy, et al., Kramer et al., and Rouch, et al., this study did not find a significant difference in the rates of renal dysfunction, or mucositis between our PO and IV cohorts [9-11]. As well, no difference in the rates of neurologic complications, or hypernatremia were seen.

Gastrointestinal intolerance is known to be associated with PO sodium bicarbonate. Therefore, the higher rates of nausea and emesis observed in our PO cohort are not surprising. These adverse effects, however, rarely led to administration issues such as refusal of doses or a need to change sodium bicarbonate formulation. Both Kramer, et al., and Rouch et al. found higher rates of diarrhea in their cohorts receiving enteral urine alkalinizing therapy $[10,11]$. In our study, the rates of diarrhea were similar between our cohorts. This discrepancy may be explained by the use of acetazolamide and higher doses of PO sodium bicarbonate administered by Kramer, et al [10]. 
This study has several limitations, most notably the retrospective design which carries the potential for missing data due to incomplete documentation in patient charts. To address such limitations, MTX clearance was defined by three different end points which increases the variability in time to MTX clearance per cycle. However, this is unlikely to impact the interpretation of the results as both cohorts had similar proportions of HDMTX cycles that met each of the three endpoints. We were also unable to control for clinician preference for adjustment of sodium bicarbonate doses and IV fluid rates leading to higher variability in the supportive therapy given during HDMTX cycles. Lastly, the use of a convenience sample may not have been sufficient to identify a statistical difference in our primary outcome. To confirm the results of this study and better support the limited literature that exists regarding PO HDMTX urine alkalinization strategies, a prospective, randomized trial design should be a consideration for future research.

While the shortage of IV sodium bicarbonate has been resolved, ongoing use of PO sodium bicarbonate for HDMTX urine alkalinization has benefit. Enteral sodium bicarbonate is significantly more cost effective than IV sodium bicarbonate. In a cost analysis performed (based on our study results), the approximate cost of sodium bicarbonate and IV fluids (excluding bolus doses) for a mean cycle duration of 105 hours and 112.6 hours for the IV and PO protocols, respectively, was \$795 CAD with the IV protocol and \$35 CAD with the PO protocol. Finally, the use of routine PO sodium bicarbonate offers the potential for initiating urine alkalinization therapy as an outpatient. This allows for shortening hospital admissions, or transitioning HDMTX administration to an ambulatory setting. Ambulatory HDMTX administration has been successfully described by Pampin et al. in a single-center prospective study [15] and Bernard et al. in a single-center retrospective review [16].

\section{Conclusion}

The results of this study indicates that the AHS PO sodium bicarbonate protocol was no different in time to MTX clearance or rates of increased serum creatinine compared to IV sodium bicarbonate. Rates of nausea and emesis occurred more frequently with the PO protocol but rarely led to administration difficulties such as refused doses or need to change sodium bicarbonate formulation. The AHS PO sodium bicarbonate protocol may offer a feasible alternative to IV sodium bicarbonate for HDMTX urine alkalinization.

\section{Declarations}

Funding: No funding was received for the completion of this study.

Conflicts of interest: The authors have no financial or non-financial interests to disclose.

Ethics Approval: Ethics approval was obtained from The Health Research Ethics Board of Alberta Cancer Committee (HREBA-CC).

Consent to participate: A waiver of consent to participate was obtained. 
Consent to publish: A waiver of consent to publish was obtained.

Availability of data and material: The authors maintain control of all the data used for the completion of this study. Anonymized data can be made available upon request.

Code availability: N/A

Acknowledgements: The authors would like to thank Matthew Newman, PharmD, BCOP and The Johns Hopkins Hospital Department of Pharmacy, Weinberg Oncology Division for their assistance with the study. We would also like to thank Melanie Varghese, BSc Pharm; Tyler Moore, BSc Pharm; Deonne Dersch-Mills, BSc Pharm, ACPR, PharmD; Ashley Yim; Eric Duong; Alexandra Yuriyivna Spirkina; and Jessica Kim for their contributions.

Author information:

Pharmacy Services, University of Alberta Hospital/Stollery Children's Hospital/Mazankowski Alberta Heart Institute, Edmonton, AB, Canada

Rachel D Heisler (https://orcid.org/0000-0001-5577-8854)

Pharmacy Services, Alberta Health Services, AB, Canada

Jordan J Kelly (https://orcid.org/0000-0001-8953-5252), Jennifer C. Jupp (https://orcid.org/0000-00025653-7312)

Faculty of Pharmacy and Pharmaceutical Sciences, University of Alberta, Edmonton, AB, Canada Sara Abendinzadegan Abdi

Pharmacy Services, Foothills Medical Centre, Alberta Health Services, Calgary, AB, Canada Jennifer L Hawker, Leanne G Fong

Pharmacy Services, Peter Lougheed Centre, Alberta Health Services, Calgary, AB, Canada Janet L Quon, Josee Z Rioux

\section{Authors' contributions:}

Conceptualization: Jennifer C. Jupp

Methodology: Jennifer C. Jupp, Jennifer L. Hawker, Leanne G. Fong, Josee Z. Rioux, Janet L. Quon, Jordan J. Kelly

Project administration \& supervision: Jennifer C. Jupp 
Data collection: Jordan J. Kelly

Data analysis: Rachel D. Heisler

Writing - original draft: Rachel D. Heisler, Sara Abedinzadegan Abdi

Writing - review \& editing: Rachel D. Heisler, Sara Abedinzadegan Abdi, Jennifer C. Jupp, Jennifer L. Hawker, Leanne G. Fong, Josee Z. Rioux, Janet L. Quon.

\section{References}

1. Howard SC, McCormick J, Pui C, et al (2016) Preventing and managing toxicities of high-dose methotrexate. Oncologist 21:1471-1482

2. Widemann BC, Adamson PC (2006) Understanding and managing methotrexate nephrotoxicity. Oncologist 11:694-703

3. Drost SA, Wentzell JR, Giguere P, et al (2017) Outcomes associated with reducing the urine alkalinization threshold in patients receiving high-dose methotrexate. Pharmacotherapy 37(6):684691

4. Alrabiah Z, Luter D, Proctor A, et al (2015) Substitution of sodium acetate for sodium bicarbonate for urine alkalinization in high-dose methotrexate therapy. AM J Health-Syst Pharm 72(15):1932-1934

5. Sterrett SP, Penniston KL, Wolf JS, et al (2008) Acetazolamide is an effective adjunct for urinary alkalinization in patients with uric acid and cystine stone formation recalcitrant to potassium citrate. Urology 72:278-281

6. Shamash J, Earl H, Souhami R (1991) Acetazolamide for alkalinization of urine in patients receiving high-dose methotrexate. Cancer Chemother Pharmacol 25(2):150-151

7. Whiteside H, Gandhi A, Ajebo G, et al (2018) When baking soda goes on shortage: urine alkalinization with acetazolamide and oral sodium bicarbonate. Ann Pharmacother 52(3):297-298

8. Cohen B, Laish I, Brosh-Nissimov T, et al (2013) Efficacy of urine alkalinization by oral administration of sodium bicarbonate: a prospective open-label trial. Am J Emerg Med 31:1703-1706

9. Roy AM, Lei M, Lou U (2019) Safety and efficacy of a urine alkalinization protocol developed for high-dose methotrexate patients during intravenous bicarbonate shortage. J Oncol Pharm Practice 25(8):1860-1866

10. Kramer E, Filtz M, Pace M (2020) Evaluation of methotrexate clearance with an enteral urine alkalinization protocol for patients receiving high-dose methotrexate. J Oncol Pharm Practice. https://doi.org/10.1177/1078155220908946

11. Rouch JA, Burton B, Dabb A, et al (2017) Comparison of enteral and parenteral methods of urine alkalinization in patients receiving high-dose methotrexate. J Oncol Pharm Practice 23(1):3-9

12. Diachinsky M, Tran T, Jupp J, et al (2020) Oral sodium bicarbonate protocol for high-dose methotrexate urine alkalinization: A pediatric experience. J Oncol Pharm Practice. 
https://doi.org/10.1177/1078155220915769

13. Visage R, Kaiser N, Williams M, et al (2019) Oral method of urinary alkalinization for high-dose methotrexate administration: Alternatives to intravenous sodium bicarbonate during a critical drug shortage. J Pediatr Hematol Oncol 41(5):371-375

14. Reed DR, Pierce EJ, Sen JM, et al (2019) A prospective study on urine alkalinization with an oral regimen consisting of sodium bicarbonate and acetazolamide in patients receiving high-dose methotrexate. Cancer Manag Res 11:8065-8072

15. Pampin R, Lebeaga $Y$, Rodriguez B, et al (2019) Experience with ambulatory high-dose methotrexate administration as CNS prophylaxis in patients with non-Hodgkin lymphoma. J Oncol Pharm Practice 26(3):549-555

16. Bernard S, Hachon L, Diasonama J, et al (2021) Ambulatory high-dose methotrexate administration as central nervous system prophylaxis in patients with aggressive lymphoma. Ann Hematol. https://link.springer.com/article/10.1007/s00277-020-04341-7

\section{Tables}

Table 1: Sodium Bicarbonate Treatment Protocol

\begin{tabular}{|lll|}
\hline & IV cohort & PO cohort \\
\hline $\begin{array}{l}\text { Routine Sodium } \\
\text { Bicarbonate Dosing }\end{array}$ & $100 \mathrm{mmol} / \mathrm{L}$ in D5W + & $1300-1500 \mathrm{mg} \mathrm{PO} \mathrm{q6h,} \mathrm{and}$ \\
\hline $\begin{array}{l}\text { As Needed Sodium } \\
\text { Bicarbonate Dosing for } \\
\text { urine pH } \geq 7\end{array}$ & $20 \mathrm{mmol} / \mathrm{L} \mathrm{KCl}$ at $200 \mathrm{~mL} / \mathrm{hr}$ & lactated Ringer's at $2 \mathrm{~mL} / \mathrm{kg} / \mathrm{hr}$ \\
\hline $\begin{array}{l}\text { Escalation of Routine } \\
\text { Sodium Bicarbonate } \\
\text { Dosing }\end{array}$ & $\begin{array}{l}\text { Increase in sodium bicarbonate } \\
\text { infusion rate as per treating } \\
\text { clinician }\end{array}$ & $\begin{array}{l}\text { Increase to } 6500 \mathrm{mg} \text { PO q6h if } \\
\text { PRN dose x2 unsuccessful, then }\end{array}$ \\
\hline
\end{tabular}

$84 \mathrm{mg}$ of sodium bicarbonate $=1 \mathrm{mmol}$ of sodium bicarbonate. Lactated Ringer's provides $28 \mathrm{mmol}$ sodium bicarbonate per liter.

Table 2: Baseline Characteristics 


\begin{tabular}{|c|c|c|c|}
\hline & IV Cohort & PO Cohort & P Value \\
\hline Patients: & $N=40$ & $N=44$ & \\
\hline Age (years); mean ( $\pm S D$ ) & $50.9( \pm 16.3)$ & $51.6( \pm 15.4)$ & 0.83 \\
\hline Male; n (\%) & $30(75)$ & $32(73)$ & 0.4 \\
\hline \multicolumn{4}{|l|}{ Diagnosis; n (\%) } \\
\hline Diffuse Large B-cell Lymphoma & $18(45)$ & $19(43)$ & 0.66 \\
\hline CNS Lymphoma & $11(28)$ & $12(27)$ & 0.68 \\
\hline Burkitt Lymphoma & $7(18)$ & $3(7)$ & 0.24 \\
\hline Acute Lymphoblastic Leukemia & $3(8)$ & $4(9)$ & 0.66 \\
\hline Osteosarcoma & $1(3)$ & $1(2)$ & 0.96 \\
\hline Other & 0 & $5(11)$ & 0.02 \\
\hline HDMTX Cycles: & $N=84$ & $N=78$ & \\
\hline Methotrexate Dose $\left(\mathrm{mg} / \mathrm{m}^{2}\right)$; mean $( \pm \mathrm{SD})$ & $3978( \pm 2509)$ & $3470( \pm 1090)$ & 0.09 \\
\hline Courses of HDMTX; median (range) & $2(1-7)$ & $2(1-9)$ & 0.82 \\
\hline Serum Creatinine $(\mu \mathrm{mol} / \mathrm{L}) ;$ mean $( \pm S D)$ & $73( \pm 20)$ & $72( \pm 23)$ & 0.82 \\
\hline
\end{tabular}

Diagnosis other: mantle cell lymphoma, follicular lymphoma, biphenotypic leukemia, plasmablastic lymphoma, peripheral T-cell lymphoma. SD: standard deviation; HDMTX: high-dose methotrexate; CNS: central nervous system

\section{Table 3: Time to Methotrexate Clearance}

\begin{tabular}{|llll|}
\hline & $\begin{array}{l}\text { IV Cohort } \\
(\mathbf{N}=\mathbf{8 4})\end{array}$ & $\begin{array}{l}\text { PO Cohort } \\
\mathbf{( N = 7 8 )}\end{array}$ & P Value \\
\hline Time to MTX Clearance (hours); mean ( $\mathbf{N S D})$ & $91.6( \pm 35.4)$ & $95.8( \pm 44)$ & 0.5 \\
\hline Primary Outcome Endpoint Met; $\mathbf{n}(\%)$ & & & \\
\hline Serum methotrexate level $\leq \mathbf{0 . 0 5} \boldsymbol{\mu \mathrm { mol } / \mathrm { L }}$ & $59(70)$ & $55(71)$ & 0.97 \\
\hline First leucovorin $\mathbf{5}$ mg dose & $11(13)$ & $16(21)$ & 0.21 \\
\hline Discharge & $14(17)$ & $7(9)$ & 0.15 \\
\hline
\end{tabular}

SD: standard deviation; IV: intravenous; PO: oral; MTX: methotrexate; HDMTX: high-dose methotrexate 
Table 4: Sodium Bicarbonate, Fluid and Leucovorin Doses

\begin{tabular}{|c|c|c|c|}
\hline & $\begin{array}{l}\text { IV Cohort } \\
(\mathrm{N}=84)\end{array}$ & $\begin{array}{l}\text { PO Cohort } \\
(N=78)\end{array}$ & $\begin{array}{l}\mathrm{P} \\
\text { Value }\end{array}$ \\
\hline $\begin{array}{l}\text { Total Sodium Bicarbonate Dose Administered During HDMTX } \\
\text { Cycle (mmol); mean ( } \pm \text { SD) }\end{array}$ & $\begin{array}{l}1984( \pm \\
850)\end{array}$ & $\begin{array}{l}992( \pm \\
432)\end{array}$ & $<0.01$ \\
\hline $\begin{array}{l}\text { HDMTX Cycles Requiring as Needed Sodium Bicarbonate Bolus } \\
\text { Doses; n (\%) }\end{array}$ & $14(17)$ & $37(47)$ & $<0.01$ \\
\hline $\begin{array}{l}\text { Total Volume of Fluid Administered During HDMTX Cycle }(\mathrm{mL}) \text {; } \\
\text { mean ( }( \pm S D)\end{array}$ & $\begin{array}{l}19692( \pm \\
8574)\end{array}$ & $\begin{array}{l}17548( \pm \\
7574)\end{array}$ & 0.09 \\
\hline Number of Leucovorin Doses $(n)$; mean ( $\pm S D$ ) & $11.7(6.4)$ & $12.4(6)$ & 0.47 \\
\hline
\end{tabular}

SD: standard deviation; IV: intravenous; PO: oral; HDMTX: high-dose methotrexate

Table 5: Change in Serum Creatinine and Adverse Events Experienced per HDMTX Cycle

\begin{tabular}{|c|c|c|c|}
\hline & $\begin{array}{l}\text { IV } \\
\text { Cohort } \\
(\mathrm{N}=84)\end{array}$ & $\begin{array}{l}\text { PO } \\
\text { Cohort } \\
(N=78)\end{array}$ & $\begin{array}{l}\mathrm{P} \\
\text { Value }\end{array}$ \\
\hline Increase in SCr by greater than $25 \%$ from baseline; $\mathrm{n}(\%)$ & $10(12)$ & $4(5)$ & 0.49 \\
\hline $\begin{array}{l}\text { Number of HDMTX Cycles Experiencing at Least One Adverse } \\
\text { event; } n(\%)\end{array}$ & $29(35)$ & $43(55)$ & $<0.01$ \\
\hline Mucositis & $14(17)$ & $14(18)$ & 0.83 \\
\hline Neurologic complications n (\%) & $4(5)$ & $4(5)$ & 0.91 \\
\hline Confusion & $4(5)$ & $3(4)$ & 0.77 \\
\hline Somnolence & $1(1)$ & $2(3)$ & 0.52 \\
\hline Seizure & 0 & $1(1)$ & 0.3 \\
\hline Nausea; n (\%) & $12(14)$ & $27(35)$ & $<0.01$ \\
\hline Emesis; n (\%) & $3(4)$ & $14(18)$ & $<0.01$ \\
\hline Diarrhea; n (\%) & $1(1)$ & 0 & 0.33 \\
\hline Hypernatremia; n (\%) & $3(4)$ & $2(3)$ & 0.71 \\
\hline
\end{tabular}

IV: intravenous; PO: oral; SCr: serum creatinine 
Figures

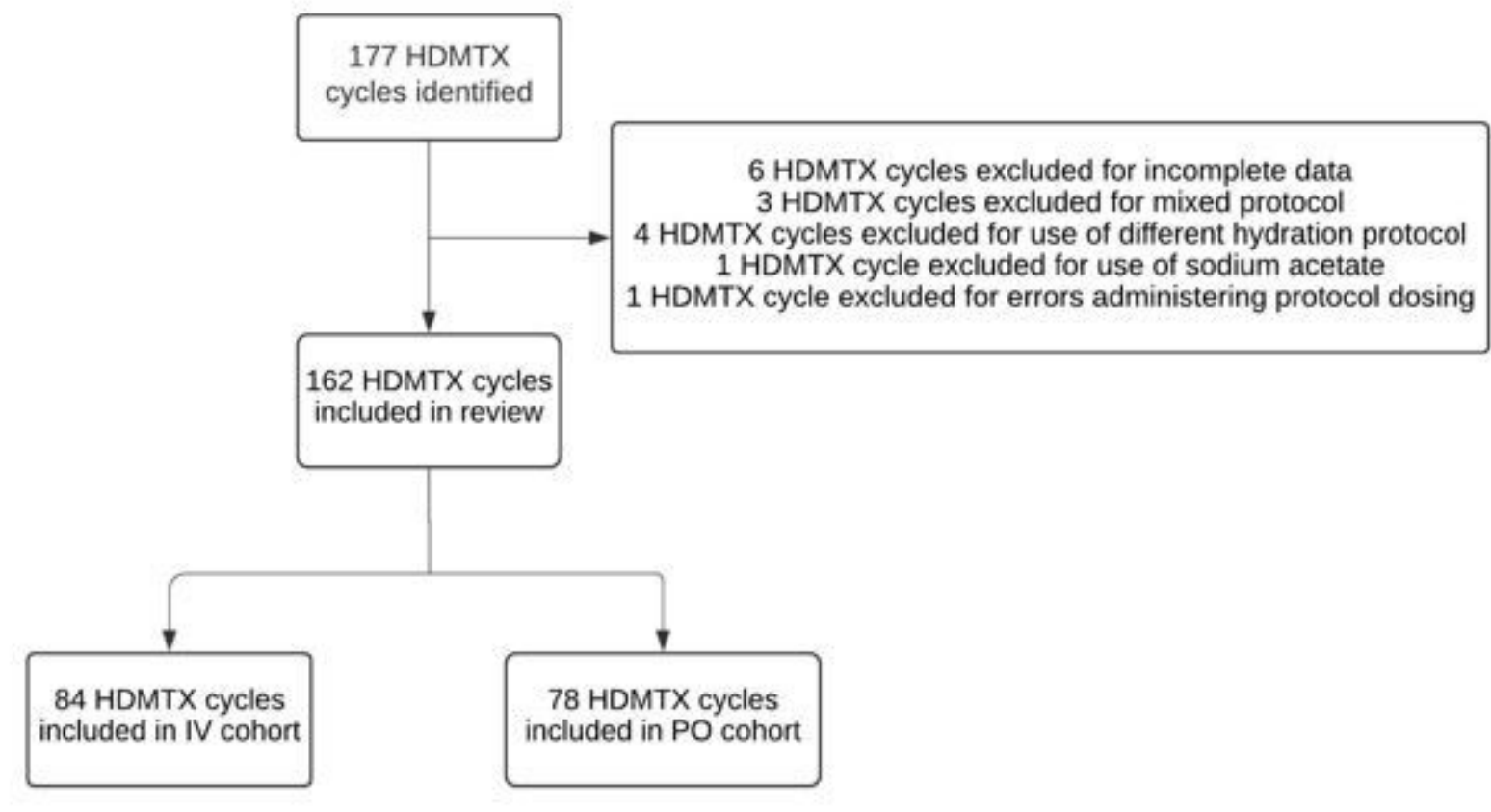

Figure 1

Study Profile. Disposition of high dose methotrexate cycles reviewed for study inclusion. HDMTX: highdose methotrexate; PO: oral; IV: intravenous

\section{Supplementary Files}

This is a list of supplementary files associated with this preprint. Click to download.

- Appendix.docx 